\section{Summary and Introduction}

The $28 \mathrm{GeV}$ proton synchrotron (PS) forms part of the chain of LE? injectors with the rôle to accelerate positrons and electrons from $0.6 \mathrm{GeV}$, the operating energy of the Electron/Positron Accumulator (EPA), to $3.5 \mathrm{GeV}$, the positron/electron injection energy of CERN's $400 \mathrm{GeV}$ proton synchrotron (SPS) which then accelerates the particles to $20 \mathrm{GeV}^{1}$ ).

The PS used as an electron synchrotron has to supply the SPS with 8 bunches at an intensity of $\mathrm{N}_{\mathrm{b}}=8 \times 10^{9}$ particles per bunch. Bunch length and energy spread must be chosen carefully to avoid transverse and longitudinal single-bunch instabilities (turbulence) associated with high peak currents in the SPS.

Analysis of the beam dynamics in the combined function lattice of the PS shows that this requirement can be fulfilled if the damping-partition numbers are changed. A Robinson Wiggler ${ }^{2}$ is proposed for this purpose and a new additional RF system is needed to produce the required bunch dimensions in langitudinal phase space.

\section{Beam Requirements}

The maximum bunch length allowed at injection in the SPS is derived from two conditions:

(i) Longitudinal matching:

$$
\frac{\sigma_{\varepsilon} / E}{\sigma_{s}}=\frac{Q_{s}}{R \alpha}
$$

where $\sigma_{E} / E=$ relative rms bunch energy spread

$$
\begin{aligned}
& \sigma_{\mathrm{S}}=\text { mms bunch leagth } \\
& \mathrm{Q}_{\mathrm{S}}=\text { synchrotron tune } \\
& \mathrm{R}=\text { mean radius }
\end{aligned}
$$$$
\alpha=\text { momentum compaction factor }
$$

(ii) Reasonable ratio between the SPS bucket half height $\Delta E$ and the bunch height $\sigma_{\varepsilon}$ :

$$
\Delta \mathrm{E} / \sigma_{\varepsilon} \geqslant \mathrm{n}
$$

where $\mathrm{n}$ is a number larger than 2.5 . Combining conditions ( 1 ) and (2) leads to:

$$
\sigma_{s} \leqslant \frac{\lambda_{r f}}{n \pi}\left[1-\left(\frac{\pi}{2}-\phi_{s}\right) \operatorname{tg} \phi_{s}\right]^{\frac{1}{2}}
$$

where $\lambda_{\mathrm{r} f}=2 \pi \mathrm{R} / \mathrm{h}=1.5 \mathrm{~m}, \mathrm{~h}=$ harmonic number and $\phi_{\mathrm{s}}=$ stable phase angle.

The corresponding upper limit of $\sigma_{s}$ versus $\sigma_{E} / E$ is plotted in fig. 1 for $n=2.6$, (Long. acceptance).

The relative rms energy spread necessary to inject above the turbulence threshold in the SPS must satisfy:

(i) for longitudinal curbulence ${ }^{3}$ )

$$
\left(\frac{\sigma_{\varepsilon}}{E}\right)_{/ 1}^{2} \geqslant \frac{e c}{(2 \pi)^{3 / 2}} \frac{N_{b}|Z / n|}{\sigma_{s} \alpha E / e}
$$

where $Z / n$ is the wall impedance, $c$ the speed of light, e the electron charge.

(ii) for transverse turbulence ${ }^{4}$ ) 5 )

$$
\left(\frac{\sigma_{c}}{E}\right)_{\perp} \geqslant \frac{K N_{b}}{F\left(\sigma_{S}\right) R a E / e}
$$

where $K=2.27 \times 10^{-3}$ and $F\left(\sigma_{S}\right) \simeq 18.1 \sigma_{S}$ for bunches longer than $7 \mathrm{~cm}$.

Fig. I shows conditions ( 3$),(4)$ and (5) where the forbidden regions are shaded. It can be seen that transverse stability imposes more stringent constraints than longitudinal stability. A working point (Ej) with a small energy spread and a long bunch length is chosen because this minimizes the wiggler strength and the required $R F$ voltage in the PS. Its coordinates define the longitudinal beam dimensions at PS ejection:

$$
\sigma_{S}=.16 \mathrm{~m} \text { and } \sigma_{E} / \mathrm{E}=10^{-3}
$$

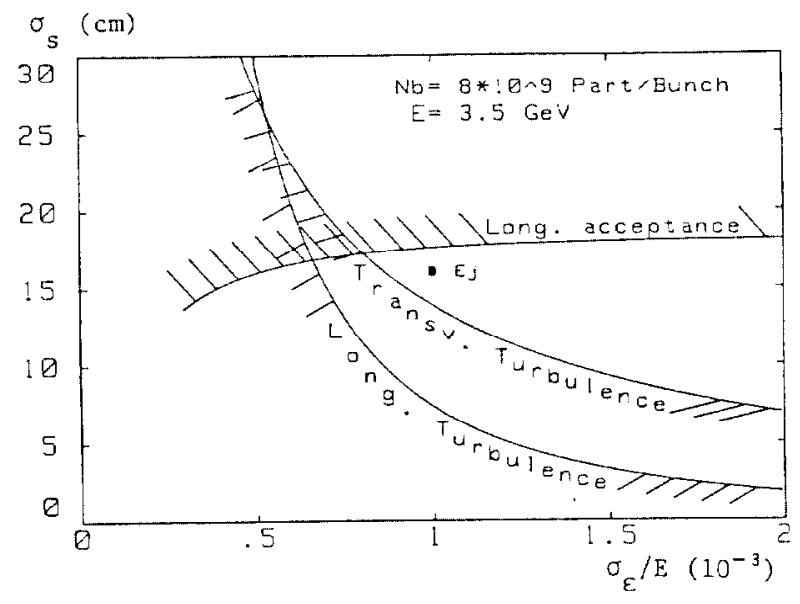

Fig. 1 Constraints at SPS injection (shaded) and location of the ejection point $\mathrm{F}_{\mathrm{j}}$.

The maximum transverse emittance is limited by the acceptance of the SPS $\mathrm{e}^{-}$injection channel to: $\varepsilon_{\mathrm{x}}(1 \sigma)=\varepsilon_{\mathrm{y}}(1 \sigma)=.16 \pi \cdot \mathrm{mm} \cdot \mathrm{mrad}$ where $x, y$ stand for the horizontal and vertical plane, respectively.

Similarly, EPA supplies the PS with properly prepared bunches $\left(\sigma_{\mathrm{s}}=.26 \mathrm{~m}\right.$ and $\left.\sigma_{\varepsilon} / \mathrm{E}=6.1 \times 10^{-4}\right)$ so that injection at $0.6 \mathrm{GeV}$ is performed above the longitudinal and transverse turbulence thresholds.

\section{Merhods of acceleration}

The present PS, with its natural longitudinal damping partition number $J_{\varepsilon}=4$ would provide at $3.5 \mathrm{GeV}$ a beam with relative rms energy spread at equilibrium of $\sigma_{E} / E=.25 \times 10^{-3}$, obtained in about 40 ms on the flat-top. With a typical acceleration cycle as shown in fig.2 the horizontal emittance would be $\epsilon_{X}(1 \sigma)=.6 \pi \cdot \mathrm{mm} \cdot \mathrm{mrad}$ at the beginning of the $3.5 \mathrm{GeV}$ flat-top and twice as big $20 \mathrm{~ms}$ later, due to the strong horizontal natural antidamping.

The equilibrium energy spread of the beam is only determined by $\mathrm{J}_{\varepsilon}$ and scales as $\mathrm{J}_{\varepsilon}{ }^{-1 / 2}$. In order to reach $\sigma_{\varepsilon} / E=10^{-3}$ (point $E j$ ) J J must be reduced. A lower value of $\mathrm{J}_{\varepsilon}$ also helps in the horizontal plane to reduce the emittance growth. Robinson wiggler magnets will be used to adjust the value of $\mathrm{J}_{E}$. Fine adjustments will be made by changing the mean radial position ${ }^{6}$ ). Two approaches are proposed for obtaining the proper bunch dimensions: bunch expansion or bunch-length compression 7 ) 8 ). 
(i) For bunch expansion a $114 \mathrm{MHz}(h=240) \mathrm{RF}$ system, providing 1 MV of peak RF voltage, is used to accelerate the beam from injection to $3.5 \mathrm{GeV}$. The required longitudinal and transverse beam dimensions are obtained through adjustment of $J_{\varepsilon}$ to the value of .16 by means of two Robinson wiggler magnets.

The choice of the RF frequency is a compromise. A lower frequency would need higher voltage to produce the short bunch length required by SPS. A higher frequency would not provide the bunch area needed for stability with an acceptable quantum life time. The harmonic number 240 is chosen hecause it allows a variety of bunch numbers: $4,6,8,12,16$ and 20 .

(ii) The second method - bunch-length compression makes use of the existing $R F$ system tuned to $7.6 \mathrm{MHz}$. Acceleration with this low-frequency system and with a modest wiggler strength $\left(\mathrm{J}_{\varepsilon}=3.1\right)$ results in a correct longitudinal bunch area at $3.5 \mathrm{GeV}$ but the bunch of $\sigma_{s}=.53 \mathrm{~m}$ is too long for the SPS and the energy spread $\sigma_{\varepsilon} / E=.3 \times 10^{-3}$ is too small.

In order to compress the bunch length and to increase the energy spread, the voltage of the $114 \mathrm{MHz}$ RF system is raised from $5 \mathrm{kV}$ to 1 MV adiabatically but fast compared to the radiation damping time ( $220 \mathrm{~ms}$ ) so that the bunch area is conserved. The $7.6 \mathrm{MHz}$ RF system cannot be used for bunch compression because all the available voltage is just sufficient to compensate for the synchrotron radiation loss (.2 MeV) at $3.5 \mathrm{GeV}$.

Since $J_{x}=-.1$ the beam is still slightly antidamped in the horizontal plane (see below).

\section{Beam dynamics for bunch expansion}

\section{Longitudinal}

The behaviour of $\sigma_{E}$ is described by:

$$
\frac{d \sigma_{\varepsilon}^{2}}{d t}=\frac{2}{\tau_{E}}\left(\sigma_{\varepsilon \text { eq }}^{2}-\sigma_{E}^{2}\right)+\sigma_{\varepsilon}^{2}\left(\frac{1}{\omega_{s}} \frac{d \omega_{s}}{d t}+\frac{1}{E} \frac{d E}{d t}\right)
$$

where $\begin{aligned} \omega_{s}=\text { angular synchrotron frequency } \\ 1 / \tau_{\varepsilon}=\text { longitudinal damping rate } \\ \sigma_{\text {Eeq. }}=\text { equilibrium rms energy spread }\end{aligned}$

For the PS:

$$
\tau_{\varepsilon}=3.16 \mathrm{E}_{[\mathrm{GeV}]}^{-3} \mathrm{~J}_{\varepsilon}^{-1}
$$

and

$$
\sigma_{\varepsilon e q} \cdot[\mathrm{GeV}]=1 . .45 \cdot 1 \sigma^{-4} \mathrm{E}^{2}[\mathrm{GeV}] \mathrm{J}_{\varepsilon}^{-\frac{1}{2}}
$$

The first term in the rhs of equation (6) describes the electron behaviour associaced with quantum excitation and radiation damping, while the second one relates to the adiabatic damping similar to the proton behaviour.

If the acceleration rate and the $114 \mathrm{MHz}$ voltage program are chosen as shown in $\mathrm{fig} .2$, with $\mathrm{J}_{\varepsilon}$ set at $.16, \sigma_{E} / E$ evolves as plotted in $f i g .3$, which is the result of a numeric integration of equation (6).

The evolution of the bunch dimensions from injection to the chosen ejection working point $\mathrm{Ej}$ is plotted in the $\sigma_{S}-\sigma_{E} / E$ plane in fig.4. It can be shown that turbulence does not occur in the PS for these bunch dimensions.

\section{Transverse}

The evolution of the transverse horizontal emittance $\varepsilon_{\mathrm{xo}}(1 \sigma)$, without coupling, is described by a similar differential equation ${ }^{3}$ :

$$
\frac{d \varepsilon_{x o}}{d t}=\frac{2}{\tau_{x}}\left(\varepsilon_{x e q}-\varepsilon_{x o}\right)-\frac{1}{E} \frac{d E}{d t} \varepsilon_{x o}
$$

where

$1 / \tau_{x}=$ horizontal damping rate

$E_{x e q}$. = equilibrium horizontal emittance
For the PS: $\quad \tau_{\mathrm{x}}=3.16 \mathrm{E}_{[\mathrm{GeV}]}^{-3} \mathrm{~J}_{\mathrm{X}^{-1}}$

$$
\text { and } \quad \varepsilon_{\mathrm{xeq}} \cdot[\pi \cdot \mathrm{mm} \cdot \mathrm{mrad}]=10^{-2} \mathrm{E}^{2}[\mathrm{GeV}] \mathrm{Jx}^{-1}
$$

Again the first term in the rhs of equation ( 7 ) describes the electron behaviour associated with quantum excitation and radiation damping while the second one relates to the adiabatic damping similar to the proton behaviour.

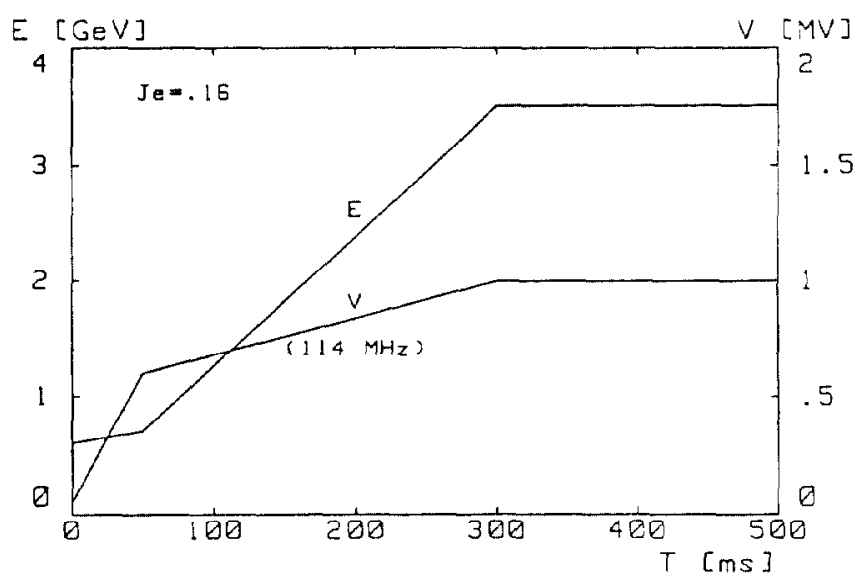

Fig. 2 Bunch expansion: Acceleration cycle and RF Voltage programme.

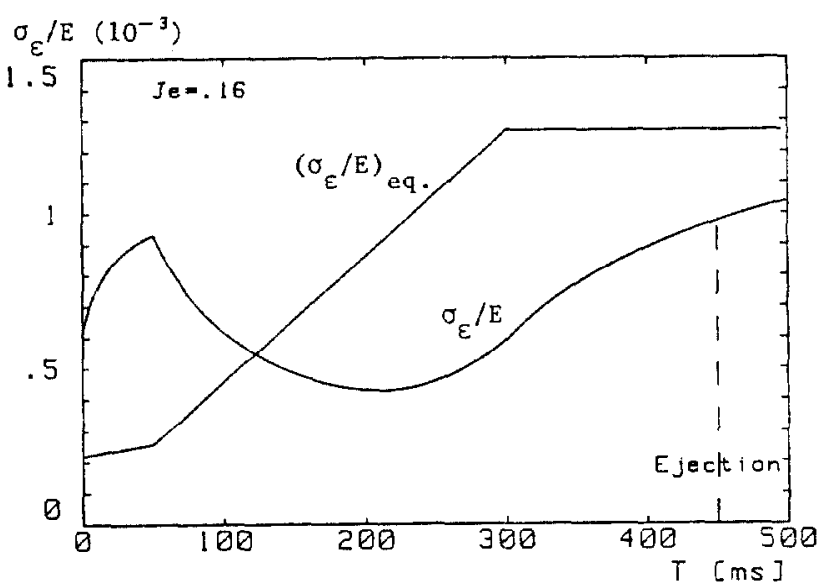

Fig. 3 Bunch expansion: Evolution of $\sigma_{E} / E$ and its equilibrium value.

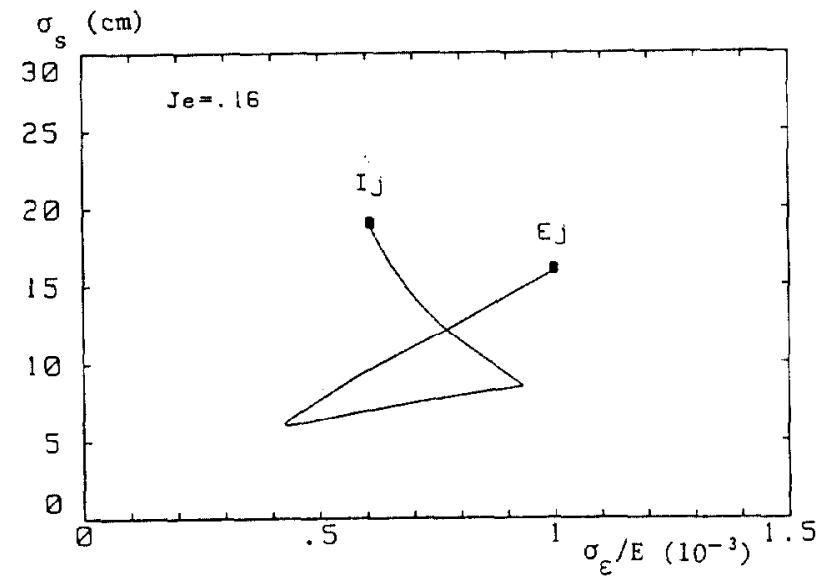

Fig. 4 Bunch expansion: Locus of longitudinal bunch dimensions from Injection ( $I j$ ) to Ejection (Ej). 
The resulting evolution of the horizontal emittance fogether with the equilibrium value is plotted in fig. 5 . The horizontal beam emittance at ejection is $\varepsilon_{x_{0}}(1 \sigma)=.045 \pi$.mm.mrad. The vertical beam emittance at extraction, only defined by parasitic coupling between horizontal and vertical motion, results, in the worst case of strong coupling, in $\varepsilon_{\mathrm{y}}(1 \sigma)=1 / 2 \varepsilon_{\mathrm{xo}}=.023 \pi \cdot \mathrm{mm} \cdot \mathrm{mrad}$.

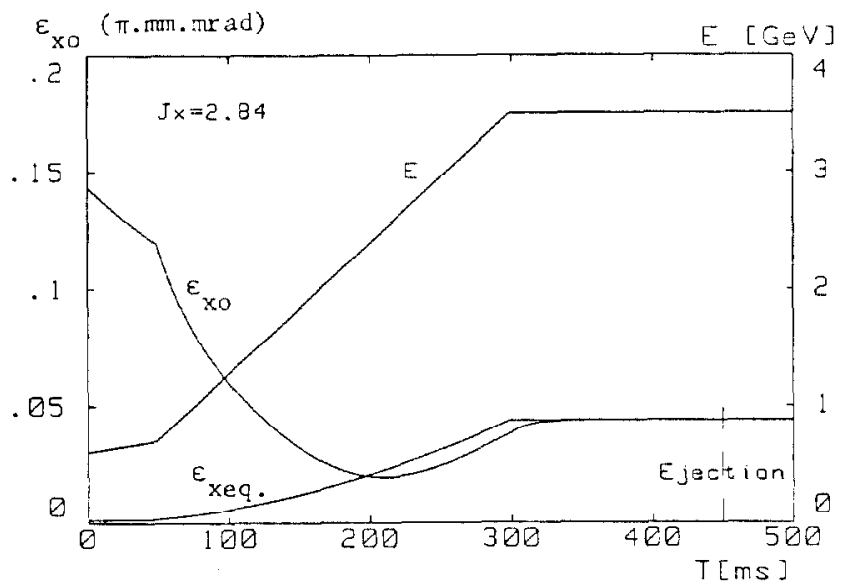

Fig. 5 Bunch expansion: Evolution of $\varepsilon_{x o}$ and its equilibrium value.

Beam dynamics for bunch-length compression

\section{Longitudinal}

The evolution of bunch dimensions in the $\sigma_{s}$ $\sigma_{E} / E$ diagram is shown in fig.6. The path from injection (point $\mathrm{Ij}$ ) to $3.5 \mathrm{GeV}$ (point $\mathrm{C}$ ) corresponds to the acceleration with the $7.6 \mathrm{MHz}$ RF system. The path from point $C$ to ejection (point $E j$ ) results from bunch length compression produced by the $114 \mathrm{MHz}$ system.

\section{Iransverse}

Fig. 7 shows the evolution of the antidamped horizontal emittance which grows to $\varepsilon_{\mathrm{xo}}(1 \sigma)=$ $.32 \pi \cdot \mathrm{mm} \cdot \mathrm{mrad}$ at the time of extraction.

In order to meet the SPS acceptance requirements strong coupling between vertical and horizontal betatron motion is used to reduce the horizontal emittance which becomes

$$
\varepsilon_{\mathrm{x}}(1 \sigma)=\varepsilon_{\mathrm{y}}(1 \sigma)<.16 \pi \cdot \mathrm{mm} \cdot \mathrm{mrad}
$$

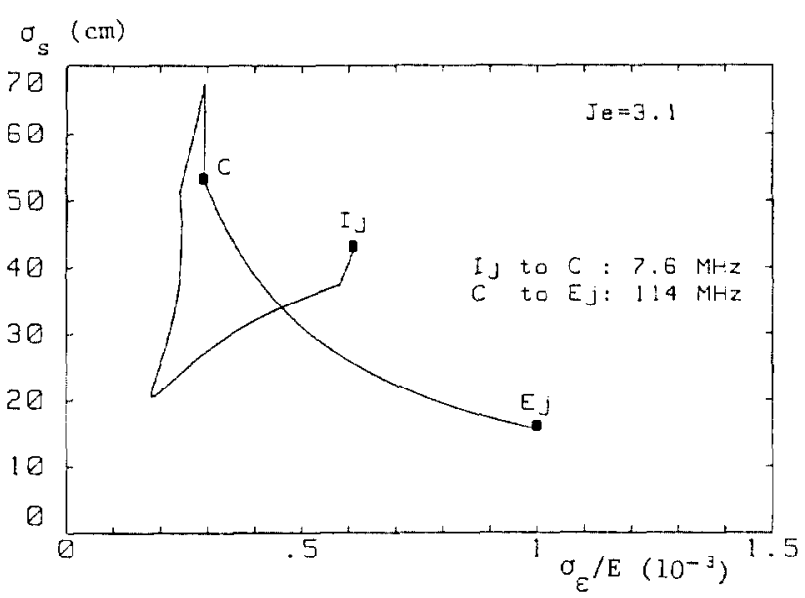

Fig. 6 Bunch-length compression: Locus of longitudinal bunch dimension during acceleration ( $f r o m$ $\mathrm{Ij}$ to $\mathrm{C}$ ) and during compression (from $C$ to $\mathrm{Ej}$ ).

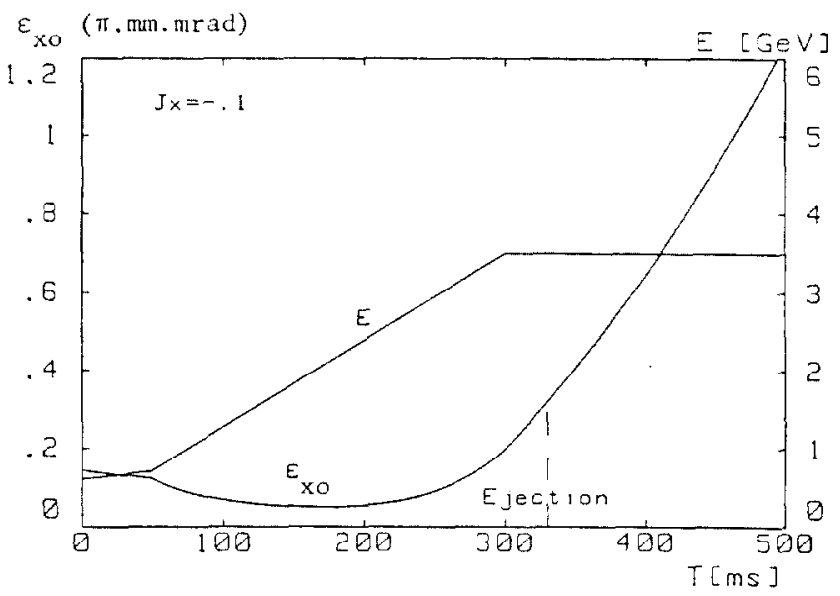

Fig. 7 Bunch-length compression: Evolution of $\varepsilon_{\mathrm{xo}}$ during the acceleration cycle.

\section{Discussion}

(i) Higher transfer energy ${ }^{8}$ to the SPS could be obtained with the bunch expansion method by making use of stronger Robinson wiggler and more $R F$ voltage at a later stage, provided that the necessary space for this additional equipment can be found in the PS ring. Bunch-length compression, however, is limited to $3.5 \mathrm{GeV}$ because the maximum available voltage of the $7.6 \mathrm{MHz}$ system is 1 imited to $220 \mathrm{kV}$.

(ii) The required $\Delta J_{\varepsilon}=-3.84$ in the bunch expansion process at $3.5 \mathrm{GeV}$ can be produced by two wigglers of $.7 \mathrm{~T}$ central field and a gradient of $8.2 \mathrm{~T} / \mathrm{m}$. Each of the wigglers is located in a short straight section between two focusing combined function magnets where the dispersion function $\left(D_{x}=\right.$ $3.05 \mathrm{~m}$ ) is constant.

(iii) As bunch-length compression uses two RF systems it is operationally more complicated than bunch expansion. Since also the transverse emittances are relatively large, bunch expansion is the preferred solution though it needs more wiggler strength. Given that the same equipment is used in both cases, no choice between the two acceleration methods needs to be made during the design stage. Experience and tests with the beam will help to select empirically the best approach.

(iv) As synchrotron radiation induced outgassing in the PS is expected to reduce significantly the electron and proton beam life time in the initial phase, special "cleaning beams" of higher intensity and energies are foreseen. Also for these beams wigglers producing $\mathrm{J}_{\mathrm{x}}>0$ are imperative to ensure beam stability on a long flat-top.

\section{References}

1. LEP Injector Study Group, this conference

2. K.W. Robinson, Phys. Rev., Vol. 111 (1958) 373

3. Y. Baconnier, J.H.B. Madsen, LEP Note 325 (1981)

4. B. Zotter, LEP Note 363 (1982)

5. J. Gareyte, SPS/DI/MST/Note 82-1 (1982)

6. H.G. Hereward, Proc. Int. Conf. on High Energy Acce1., Brookhaven (1961) 222

7. K. Hübner, J.H.B. Madsen, LEP Note 378 (1982)

8. R. Cappi, LEP Note 416 (1982)

9. W. Hardt, CERN/PS/DL/LEP/Note 80-5 (1980) 